\title{
Stahl-UHFB - Stahlbeton Verbundbauweise zur Verstärkung von bestehenden Stahlbetonbauteilen mit Ultra-Hochleistungs-Faserbeton (UHFB)
}

Die Grundidee der Stahl-UHFB - Stahlbeton Verbundbauweise besteht darin, mit einer UHFB Schicht, die in Haupttragrichtung mit Bewehrungsstäben versehen ist, Bauteile aus herkömmlichem Stahlbeton zu verstärken, indem deren Tragfähigkeit erhöht und die Dauerhaftigkeit verbessert werden. Dadurch können die bekannten Schwachstellen und Mängel der Betonbauweise eliminiert werden. Es wird ein Überblick über die StahlUHFB - Stahlbeton Verbundbauweise gegeben, indem die wesentlichen theoretischen Aspekte des Tragverhaltens von Stahl-UHFB - Stahlbeton Verbundbauteilen kurz erläutert und anhand von Anwendungen bei der Instandsetzung und Verstärkung bestehender Betonbauten illustriert werden. Schließlich werden Möglichkeiten des Einsatzes dieser neuartigen Bauweise beim Bau von neuen Tragwerken aufgezeigt.

\section{$1 \quad$ Einleitung}

Seit jeher haben neuartige Baustoffe dem konstruktiven Ingenieurbau $\mathrm{zu}$ einer Weiterentwicklung verholfen. Ultra-Hochleistungs-Faserfeinkorn-Betone (UHFB - diese werden oft zur Familie von Ultra-High Performance Concretes (UHPC) gezählt, die im Rahmen dieses Aufsatzes behandelten UHFB enthalten Stahlfasern) haben das Potenzial, als neuartige Baustoffe die etablierte Betonbauweise zu ergänzen und zu verbessern, denn UHFB zeichnet sich im Vergleich $\mathrm{zu}$ herkömmlichen zementösen Baustoffen durch vergleichsweise hohe mechanische Festigkeiten und eine sehr geringe Permeabilität aus. Der in diesem Aufsatz verwendete Begriff „Verstärkung“ umfasst die Verbesserung der Dauerhaftigkeit sowie die Erhöhung des Tragwiderstands und der Steifigkeit von Bauteilen.

Die Grundidee der Verstärkung von Bauteilen aus Stahlbeton mit UHFB besteht darin, eine 25 bis $80 \mathrm{~mm}$ starke UHFB Schicht auf Stahlbeton aufzubringen. Die Kontaktfläche wird vorgängig mit Methoden wie Hochdruckwasserstrahlen oder Sandstrahlen aufgeraut, um den notwendigen Verbund $\mathrm{zu}$ erhalten. Indem in die UHFB Schicht in Haupttragrichtung Bewehrungsstäbe von vergleichsweise kleinen Durchmessern ( 8 bis $12 \mathrm{~mm}$ ) und Abständen (30 bis $100 \mathrm{~mm}$ ) eingelegt werden, wird - in Anlehnung an den Begriff „Stahlbeton“ - der sogenannte „Stahl-UHFB“ erhalten. Die Kombination von UHFB resp. Stahl-UHFB mit Stahlbeton führt zu Verbundbauteilen gemäß Bild 1 .

Durch die Ergänzung des Stahlbetonbauteils mit StahlUHFB werden Verbundbauteile erhalten, die den Trag-
Strengthening of existing reinforced concrete elements using Ultra-High Performance Fiber Reinforced Concrete (UHPFRC) An original concept is presented for the strengthening of concrete structures. The main idea is to use Ultra-High Performance Fibre Reinforced Concrete (UHPFRC) complemented with steel reinforcing bars to strengthen those zones of the structure that are exposed to severe environmental influences and high mechanical loading. This concept combines efficiently protection and resistance properties of UHPFRC and significantly improves the structural performance of the rehabilitated concrete structure in terms of durability. Properties of UHPFRC are outlined and the essential theoretical background of structural behavior of R-UHPFRC - RC composite elements is given. This novel technology has been validated by means of field applications.

widerstand deutlich zu erhöhen vermögen sowie im Gebrauchszustand bedeutende Zugkräfte aufnehmen und so die Steifigkeit erhöhen, ohne dass es zu einer sichtbaren und für die Dauerhaftigkeit nachteiligen Rissbildung kommt. Damit können gleichzeitig tragende und flüssigkeitsdichte UHFB-Schichten auf denjenigen Bereichen aus Stahlbeton aufgebracht werden, welche durch starke Umwelteinflüsse (z.B. Einwirkung von Wasser mit/ohne Tausalzen) oder hohe mechanische Einwirkungen (z. B. infolge Verkehrslasten auf Fahrbahnplatten) stark beansprucht sind. Falls die UHFB Schicht einzig eine Schutzfunktion zu erfüllen hat, dann sind Einlagen von Bewehrungsstäben in der Regel nicht notwendig und die UHFB Schicht kann auf minimal $25 \mathrm{~mm}$ Dicke reduziert werden.

Wie für andere hochleistungsfähige Baustoffe gilt die Strategie, den UHFB nur dort einzusetzen, wo seine vor-
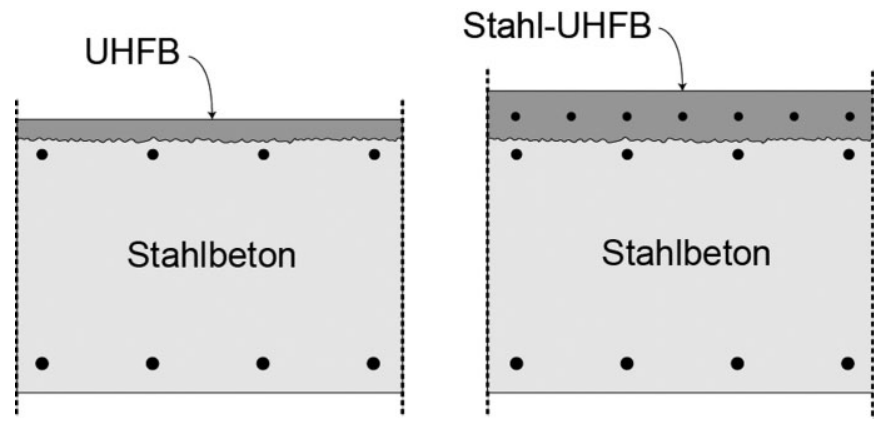

Bild 1 Grundkonfiguration für die Verstärkung von Stahlbeton mit UHFB Basic configuration for the strengthening of reinforced concrete using UHPFRC 
teilhaften Eigenschaften auch genutzt werden. Der verbleibende volumenmäßig größere Anteil des Bauteils soll in herkömmlichem Stahlbeton belassen werden. Mit der Stahl-UHFB - Stahlbeton Verbundbauweise kann die Tragfähigkeit von Tragwerken und Bauteilen erhöht und die Dauerhaftigkeit verbessert werden. Die bekannten Schwachstellen und Mängel der etablierten Betonbauweise, die auch bei Anwendung heute geltender Normenregelungen bestehen bleiben, können dadurch elegant eliminiert werden. Die Anwendung von Stahl-UHFB - Stahlbeton Verbundbauteilen zielt in erster Linie auf Platten als Bauteile des Hochbaus und von Ingenieurbauten wie Brücken.

Bei bestehenden Betonbauten besteht das Ziel und der Nutzen des Einsatzes von UHFB und Stahl-UHFB darin, sowohl die Tragfunktion (Tragwiderstand gegenüber Biegung, Schub, Ermüdung) als auch die Schutzfunktion (Abdichtung) in einem Arbeitsgang herstellen zu können, was in vielen Fällen zu einer deutlichen Reduktion der Dauer der Baustelle und damit der indirekten Kosten (Benutzerkosten) führt. Diese Bauweise bedeutet auch einen sanften Eingriff in das bestehende Tragwerk, da sein Eigengewicht nicht oder nur unwesentlich erhöht werden muss, womit Verstärkungen anderer Tragwerksteile und der Gründungen vermieden werden können.

Seit dem Sommer 1999 befasst sich die Forschungsgruppe der Autoren an der ETH Lausanne (EPFL) mit der Thematik des Einsatzes von UHFB für die Instandsetzung und Verstärkung von bestehenden Bauwerken aus Stahlbeton, insbesondere von Brïcken. Von den grundlegenden Erkenntnissen dieser Forschung ausgehend, fokussiert dieser Aufsatz fokussiert auf wesentliche Aspekte der Stahl-UHFB - Stahlbeton Verbundbauweise für den Entwurf und die Bemessung von Verstärkungen. Zudem werden bisher ausgeführte Anwendungen beschrieben.

\section{Herstellung und Eigenschaften von UHFB und Stahl-UHFB}

\subsection{Ausgangsstoffe und Zusammensetzung von UHFB}

Ultra-Hochleistungs-Faserbeton (UHFB) ist ein aus Zement, Zusatzstoffen, Gesteinskörnung, Wasser, Zusatzmittel und Fasern hergestellter Verbundwerkstoff, dessen Zugverhalten sich durch ein Verfestigungs- und Dehnungsentfestigungs-Verhalten auszeichnet, dessen charakteristischer Wert der Zylinderdruckfestigkeit nach 14 Tagen höher ist als $120 \mathrm{MPa}$ und der wegen seiner hohen Packungsdichte im Gebrauchszustand flüssigkeitsdicht ist. Die höchsten mechanischen Festigkeitseigenschaften von UHFB werden mit Stahlfasern einer Länge von 10 bis $13 \mathrm{~mm}$ und einer Dicke von etwa $0,15 \mathrm{~mm}$ mit einem Mindestfasergehalt von 3 Vol.-\% erreicht.

Die Art, Menge und Zusammensetzung der Ausgangsstoffe eines UHFB ist spezifisch für jede Sorte. UHFB wird als Fertigprodukt angeboten oder aufgrund einer Rezep- tur gemischt. Der Baustoffmarkt bietet heute mehrere UHFB-Produkte als Fertigmischungen an. UHFB kann sowohl in Form von Fertigteilen, vorwiegend für den Neubau, eingesetzt werden oder vor Ort bei Eingriffen in bestehende Tragwerke als selbstverdichtender FrischUHFB angeliefert und eingebaut werden.

Die Fasern sind ein wesentlicher Kostenpunkt und entsprechend kommt einem optimierten Fasergehalt eine große Bedeutung zu. Anhand von numerischen Simulationen und analytischen Untersuchungen wurde der Einfluss des Gehalts und der Orientierung der Stahlfasern (in der Regel: Länge 10 bis $13 \mathrm{~mm}$, Durchmesser 0,15 bis $0,2 \mathrm{~mm}$ ) auf die mechanischen Eigenschaften von UHFB unter Zugbeanspruchung ermittelt [1]. Daraus konnte abgeleitet werden, dass für die UHFB Schicht, die im Verbundquerschnitt infolge Verformungsbehinderung durch den Untergrund aus Stahlbeton durch bedeutende Zugeigenspannungen beansprucht wird, ein Mindestfasergehalt von 3 Vol.- $\%$ nicht unterschritten werden sollte, denn nur so kann das dabei erforderliche Verfestigungsverhalten des UHFB erhalten werden. Für vorwiegend druckbeanspruchte Tragwerksteile darf der Fasergehalt auch Werte im Bereich von 2 Vol. $-\%$ annehmen.

Die weitere Entwicklung des Baustoffs UHFB betrifft seine Optimierung, die in zwei Richtungen geht: Bei der Zementmatrix wird die Zementmenge (und dabei vor allem der Klinkeranteil) reduziert und durch die Beigabe von Kalksteinmehl ergänzt. Eine weitere Tendenz ist die Zugabe von schwindreduzierenden Zusatzstoffen. Bei den Fasern werden Möglichkeiten zur Substitution der Stahlfasern durch Polymerfasern untersucht. Diese Optimierungen zielen in erster Linie auf eine Reduktion der Baustoffkosten für UHFB und eine Verbesserung der Umweltverträglichkeit.

\subsection{Technologische Aspekte}

Entscheidend bei der Herstellung des selbstverdichtenden Frisch-UHFB ist der Herstellungsprozess, der je nach UHFB-Produkt unterschiedlich ist und genau eingehalten werden muss. Denn nur so kann der Frisch-UHFB mit den geforderten Eigenschaften betreffend Verarbeitbarkeit gefertigt werden. Auch konnten in letzter Zeit selbstverdichtende Frisch-UHFB Mischungen durch die Zugabe entsprechender Zusatzstoffe entwickelt werden, die nach dem Einbau auf der Baustelle ein Gefälle von bis zu $10 \%$ halten können ohne „davonzufließen“. Eine interessante, für die Qualitätskontrolle bei der Ausführung sehr wesentliche Erkenntnis ist, dass ein korrekt hergestellter Frisch-UHFB im erhärteten Zustand mit großer Wahrscheinlichkeit die geforderten mechanischen Eigenschaften aufweisen wird $[2,3]$. In Bezug auf den Eindringwiderstand von (chloridhaltigem) Wasser in UHFB haben Permeabilitätsmessungen Werte ergeben, die um etwa zwei Größenordnungen besser sind als entsprechende Permeabilitätswerte, wie sie für heute übliche, sogenannt dichte Betone gemessen werden [4, 5]. 
Die Stahlfaserspitzen an der UHFB Oberfläche korrodieren, vor allem bei Kontakt mit chloridhaltigem Wasser (vgl. Bild 6a). Diese Korrosionserscheinung ist für die Dauerhaftigkeit unbedeutend, kann aber das Aussehen ungünstig beeinflussen. Das Auftreten von „Rostpunkten“ wird durch die Oberflächenbeschaffenheit der Schalung beeinflusst. Beim Einsatz einer Schalung aus Stahl ergibt sich die stärkste und bei einer Holzschalung die geringste Bildung von „Rostpunkten“ bei Beaufschlagung der UHFB Oberfläche mit chloridhaltigem Wasser [4]. Eine auf der Baustelle hergestellte UHFB Oberfläche eines Randabschlusses, bei der die Oberfläche mit einer in die Schalung eingelegte wasserabführende Schalungsbahn bearbeitet wurde (vgl. Bild $6 \mathrm{~b}$ ), zeigt nur noch wenige „Rostpunkte“ $[2,6]$.

Beim Einsatz von UHFB auf Fahrbahnplatten von Straßenbrücken ist der Verbund zwischen UHFB und dem Fahrbahnbelag von großer Bedeutung. Bei Schichtstärken des Fahrbahnbelags von 80 bis $100 \mathrm{~mm}$ können übliche Belagssysteme eingebaut werden. Im Hinblick auf eine Reduktion der ständigen Einwirkungen bei der Verstärkung von bestehenden Betonbrückenplatten wurde eine Belagsmischung optimiert, die mit einer Schichtstärke von nur $40 \mathrm{~mm}$ eingebaut werden kann und die eine gemäß den Normen erforderliche Oberflächenhaftzugfestigkeit in Höhe von 1,0 MPa aufweist [4]. Anhand von Versuchen wurde auch die Temperatureinwirkung beim Belagseinbau auf eine UHFB Schicht experimentell und numerisch untersucht und dabei festgestellt, dass die Eigenschaften des UHFB nicht beeinträchtigt werden.

\subsection{Eigenschaften von UHFB und Stahl-UHFB}

Da bei der Erhärtung das Anmachwasser beinahe vollständig verbraucht wird und sich im dichten Gefüge der Matrix kaum noch Kapillarporen bilden, weist der Baustoff eine sehr geringe Permeabilität gegenüber Wasserund Chlorideintrag auf [7]. Das im einachsigen Zugversuch beobachtete Zugverhalten (Bild 2) zeigt nach Erreichen der sogenannten elastischen Zugfestigkeit $f_{U t e}$, bei der die Matrix seine Zugfestigkeit erreicht, eine deutliche Verfestigung (strain hardening) des Werkstoffs von $\varepsilon_{U}=1$ bis 3\% Dehnung bei Erreichen der UHFB-Zugfestigkeit von $f_{U t}=9$ bis $15 \mathrm{MPa}$ [8], gefolgt von einer ausgeprägten Dehnungsentfestigung (strain softening) bis zu einer maximalen Makrorissöffnung von $w_{u t \text {,max }}$ von etwa der halben Faserlänge, mit einer spezifischen Bruchenergie $G_{F U}$ von etwa $20 \mathrm{~kJ} / \mathrm{m}^{2}$. Der Elastizitätsmodul von UHFB beträgt $E_{U}=45$ bis $55 \mathrm{GPa}$. Die Druckfestigkeit von UHFB $f_{U c}$ beträgt mehr als $150 \mathrm{MPa}$.

Durch die Ergänzung von UHFB mit Bewehrungsstäben, die nur in Haupttragrichtung eingelegt werden, können im Verbundquerschnitt bedeutende Zugkräfte aufgenommen werden [9 bis 13]. Gerippte und glatte Bewehrungsstäbe unterschiedlicher Festigkeiten sind für die Bewehrung des UHFB geeignet. Mit Rippenstählen werden höhere Tragwiderstände erzielt. Glatte Stähle erlauben
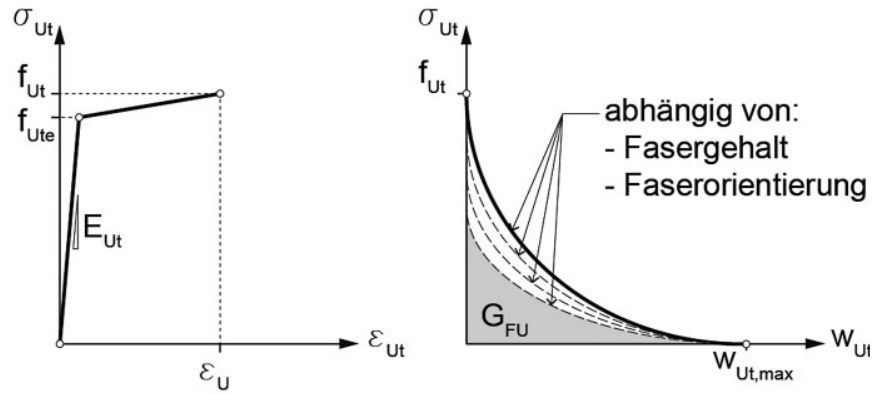

Bild 2 Stoffgesetze für UHFB unter Zugbeanspruchung Constitutive laws for UHPFRC under tensile stresses
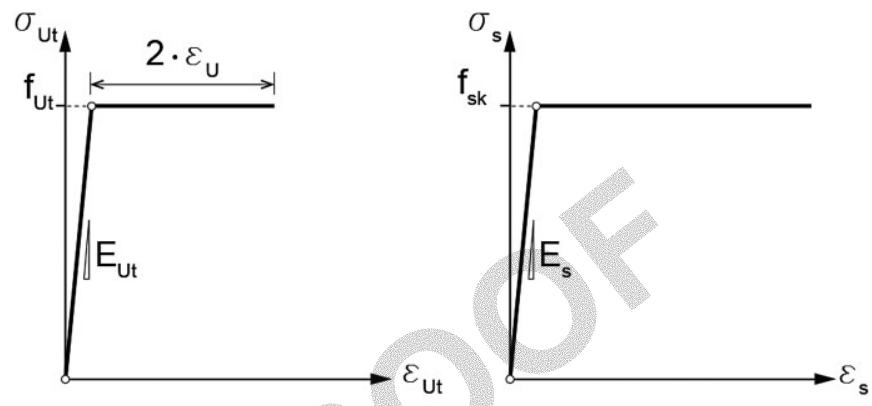

Bild 3 Stoffgesetze für UHFB und Betonstahl für Bauteile aus Stahl-UHFB unter Zugbeanspruchung $\left(\sigma_{U t}, \varepsilon_{U t}\right.$ : Zugspannung resp. Dehnung im UHFB; $\sigma_{s}, \varepsilon_{s}$ : Spannung resp. Dehnung im Betonstahl) Constitutive laws for UHPFRC and reinforcing steel for elements made in R-UHPFRC under tensile stresses $\left(\sigma_{U \mathrm{Ut}}, \varepsilon_{\mathrm{Ut}}\right.$ : tensile stress and strain respectively in the UHPFRC; $\sigma_{\mathrm{S}}, \varepsilon_{\mathrm{S}}$ : tensile stress and strain respectively in the steel rebar)

größere Gesamtverformungen. Die Kraft-VerformungsAntwort von Stahl-UHFB wird durch Superposition der Stoffgesetze UHFB und Betonstahl (Bild 3) erhalten; die „Fließspannungen“ der beiden Baustoffe treten bei einem ungefähr gleichen Dehnungswert auf $[9,10]$.

Durch die Kombination von Stahl-UHFB mit Stahlbeton kann ein beachtlicher Steifigkeitsgewinn der Tragwerksteile und somit eine Steigerung der Gebrauchstauglichkeit erreicht werden. Zudem wird die für UHFB charakteristische Streuung der mechanischen Eigenschaften, die herstellungsbedingt auf eine anisotrope Faserausrichtung zurückzuführen ist, durch die Verwendung von Stabstählen deutlich reduziert $[9,10]$.

\section{Tragfähigkeit und Bemessung von Stahl-UHFB - Stahlbeton Verbundbauteilen 3.1 Allgemeine Erkenntnisse zum Tragverhalten}

Im Verbundsystem Stahl-UHFB - Stahlbeton entstehen in der UHFB Schicht beim Erhärten Zugspannungen infolge von Schwinden und Verformungsbehinderung durch den Stahlbeton-Untergrund [14, 15]. Diese Zugspannungen nehmen je nach Einspanngrad der UHFB-Schicht Werte von $6-8 \mathrm{~N} / \mathrm{mm}^{2}$ an, die vom UHFB wegen dessen vergleichsweise hoher Zugfestigkeit und dem verfestigenden Verhalten aufgenommen werden können. Entsprechend kann davon ausgegangen werden, dass im UHFB unter 
Gebrauchsspannungen keine sichtbaren Risse auftreten. Diese Erkenntnis stützt sich auf Beobachtungen an Bauwerken und Ergebnissen von numerischen Simulationen typischer Verbundsysteme, die in Versuchen und Ausführungen realisiert und messtechnisch erfasst wurden.

Stahl-UHFB - Stahlbeton Verbundbauteile verhalten sich monolithisch. Versuche zum Langzeitverhalten haben gezeigt, dass an der Grenzschicht und an den freien Enden keine Ablöseerscheinungen auftreten. Unter Biegebruchbeanspruchung treten Risse infolge von Scherbeanspruchung zwischen den beiden Schichten erst weit nach Überschreiten des maximalen Tragwiderstands auf [16 bis 19]. Die Steifigkeit, die Tragfähigkeit und der Tragwiderstand von UHFB-Stahlbeton Verbundbauteilen werden durch das Einlegen einer Bewehrung in der UHFB Schicht erhöht. Zudem vergrößern sich die Zugverformungen im verfestigenden Bereich des UHFB $[9,17,18]$.

Das Tragverhalten von Stahl-UHFB - Stahlbeton Verbundbauteilen kann mit analytischen und numerischen Modellen vorhergesagt werden, insbesondere das zeitabhängige Verhalten [20, 21]. Das Verhalten unter Biegung wird mit einem Querschnittsmodell erfasst, das eine Erweiterung des klassischen Biegemodells für Stahlbeton ist (Bild 4) [10, 16].

\subsection{Nachweis der Tragsicherheit}

Das duktile Tragverhalten erlaubt es, den Tragwiderstand von Stahl-UHFB - Stahlbeton Verbundbauteilen nach der Plastizitätstheorie zu ermitteln. Da im Verbundquerschnitt mehrere Baustoffe beansprucht sind und um das Nachweisformat übersichtlich zu halten, wird der Bemessungswert des Tragwiderstands von verstärkten Bauteilen wie folgt ermittelt:

$$
R_{d}=\frac{R_{k}}{\gamma_{M}}
$$

Dabei wird der charakteristische Wert des Tragwiderstands mit den aktualisierten Abmessungen des bestehenden Stahlbetonbauteils und dessen Querschnittsflächen sowie mit den charakteristischen Werten der Baustoffeigenschaften ermittelt. Der Widerstandsbeiwert $\gamma_{M}=\gamma_{m}$. $\gamma_{R}$ berücksichtigt einerseits ungünstige Abweichungen der Baustoffeigenschaften vom charakteristischen Wert mit $\gamma_{m}=1,10$ (da der Tragwiderstand maßgeblich vom StahlUHFB beeinflusst ist), und andererseits die Unschärfen im Widerstandsmodell mit $\gamma_{R}=1,10$. Somit beträgt der Widerstandsbeiwert $\gamma_{M}=1,20$. Der charakteristische Wert des Biegetragwiderstands darf unter Verwendung der charakteristischen Werte der Baustoffeigenschaften mit den Modellen gemäß Bild 4 ermittelt werden. Dabei ist insbesondere bei der Konfiguration gemäß Bild 4a) nachzuweisen, dass der bestehende Betonquerschnitt, der in der Regel unverstärkt bleiben soll, die höhere Druckbeanspruchung auch aufnehmen kann. Dies ist oft der Fall, denn die Betonfestigkeit bestehender Betonbauten hat über die Jahre deutlich zugenommen.

Das Tragverhalten von Stahl-UHFB - Stahlbeton Verbundbalken unter kombinierter Einwirkung von Quer-

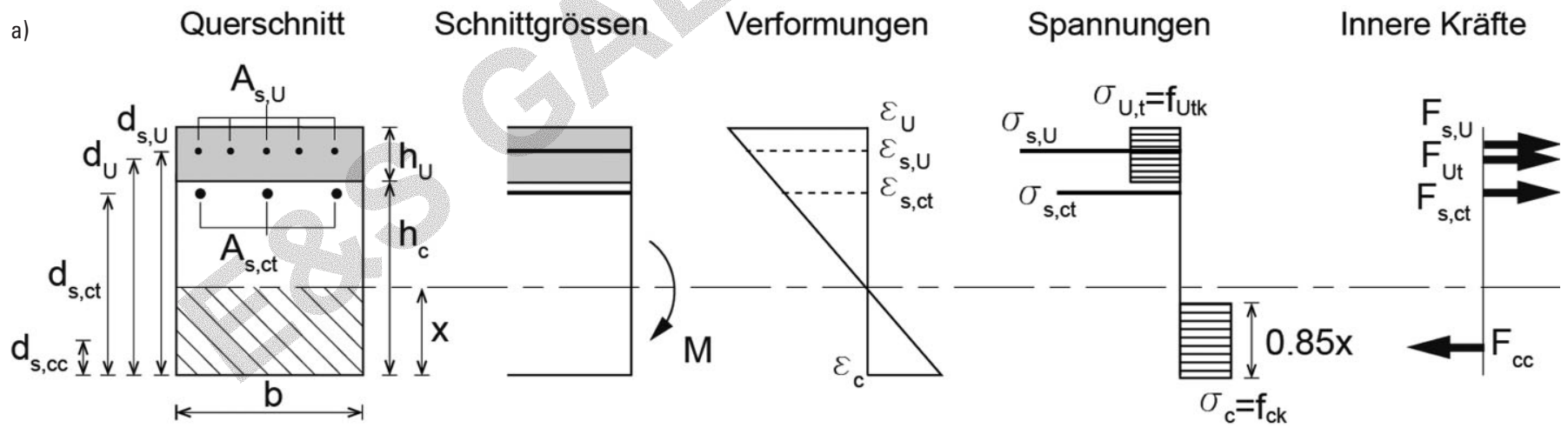

b)
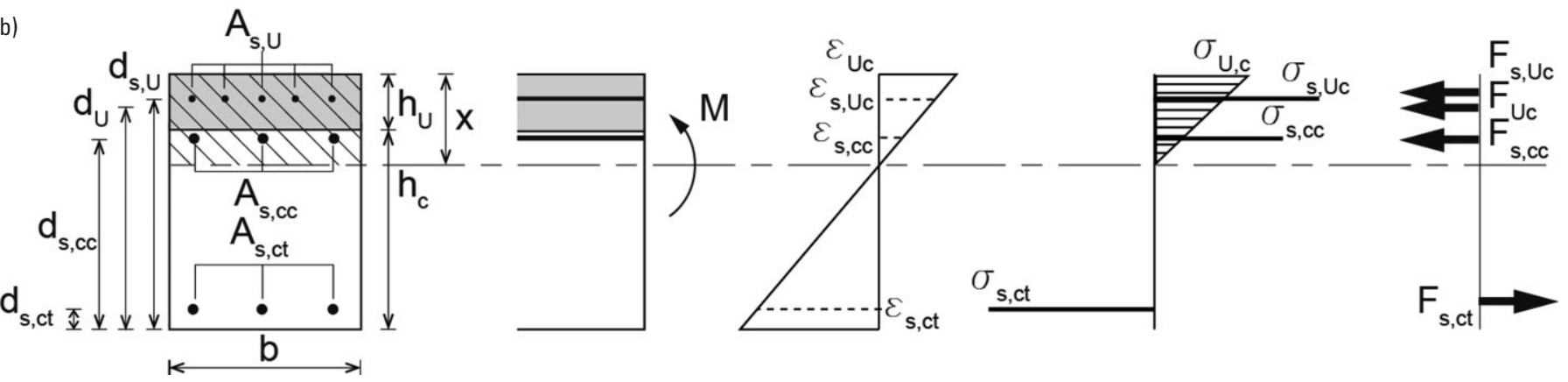

Bild 4 Biegewiderstand von Stahl-UHFB - Stahlbeton Verbundquerschnitten im Grenzzustand der Tragfähigkeit: a) für negative Momente: UHFB unter Zugbeanspruchung, b) für positive Momente: UHFB unter Druckbeanspruchung.

Ultimate bending resistance of R-UHPFRC - RC composite sections at ultimate limit state: a) negative moments with UHFPRC under tensile stresses, b) positive moments with UHPFRC under compressive stresses. 

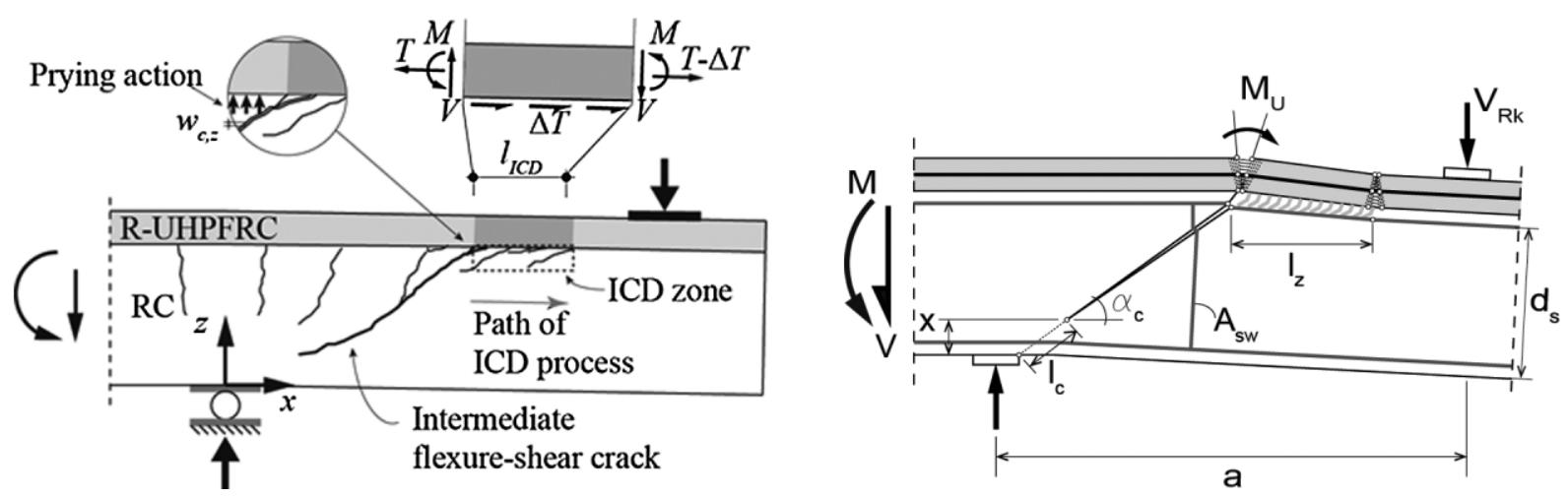

Bild 5 Stahl-UHFB - Stahlbeton Verbundträger unter Biegung und Schub: Versagensmechanismus und Modell für den Schubtragwiderstand [22, 23] R-UHPFRC - RC composite beam subjected to combined bending and shear: failure mechanism and shear resistance modell $[22,23]$

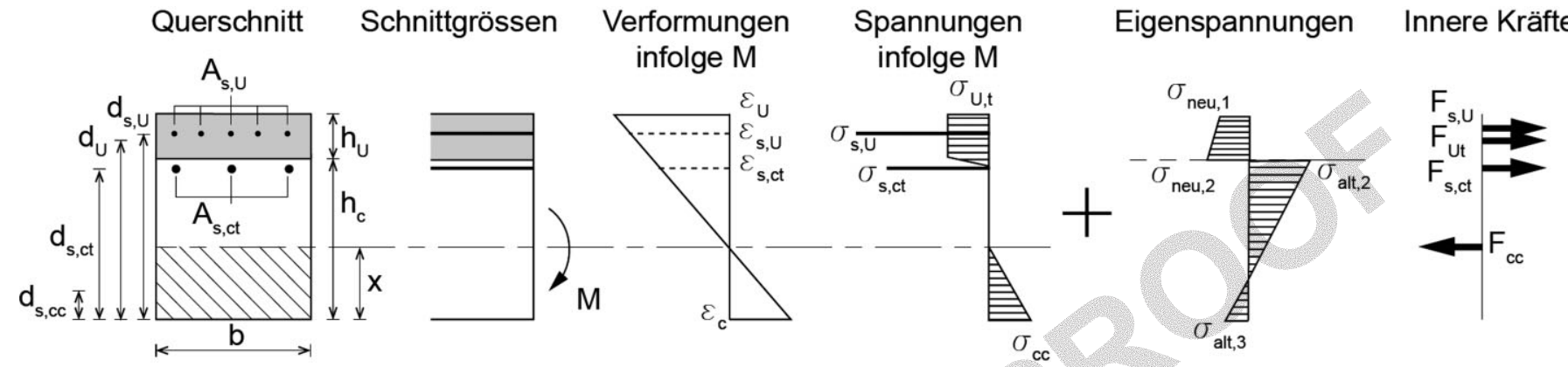

Bild 6 Spannungsermittlung im Grenzzustand der Gebrauchstauglichkeit für negative Momente: UHFB unter Zugbeanspruchung Stress determination at serviceability limit state for negative moments with UHFPRC under tensile stresses

kraft und Biegemoment wurde in einer kürzlich abgeschlossenen Dissertation untersucht [22, 23]. Anhand von Versuchen an Verbundbalken unter Schub-Biegebeanspruchung konnte zunächst festgestellt werden, dass sich in der Regel Biegemechanismen als maßgebende Versagensart bilden, obwohl Konfigurationen für den Stahlbetonteil gewählt wurden, bei denen eindeutig ein Schubbruch zu erwarten war. Die verstärkende Stahl-UHFB Schicht führt offenbar zu einer deutlich stärkeren Erhöhung der Schubtragfähigkeit als der Biegetragfähigkeit.

Bei Probekörperkonfiguration, bei denen sich ein Schubbruch einstellte, konnte beobachtet werden, dass nach dem Auftreten des geneigten Schubhauptrisses sich im Stahlbetonteil eine (unter entsprechender Rissbildung) entfestigende Zone ICD entlang der Grenzschicht zwischen Stahl-UHFB und Stahlbeton ausbildete (Bild 5). Erst nach Auftreten dieses Mechanismus konnte der Stahl-UHFB seinen Tragwiderstand entwickeln, und der Verbundträger erreichte schließlich seinen Tragwiderstand auf einem gegenüber dem unverstärkten Stahlbetonträger deutlich höheren Niveau.

Ausgehend von diesen Erkenntnissen wurden Modelle gemäß der Plastizitätstheorie hergeleitet zur Ermittlung des Tragwiderstands und der Tragwerksantwort von Stahl-UHFB - Stahlbeton Verbundträgern unter Schubbeanspruchung [23]. Es konnte gezeigt werden, dass sich der Schubtragwiderstand zusammensetzt aus der Superposition des Schubtragwiderstands des Betons im Steg entlang der geneigten Länge $l_{c}$, dem Tragwiderstand der (allenfalls vorhandenen) vertikalen Betonstahlbewehrung sowie dem Schubwiderstand $V_{R U}$ der Stahl-UHFB Schicht mit dem Zwei-Gelenk-Mechanismus entlang der Länge $l_{z}$ gemäß:

$$
V_{R U}=\frac{2 M_{U}}{l_{Z}}
$$

mit: $M_{U}$ plastisches Moment der Stahl-UHFB Schicht

Eine aktuelle Forschung betrifft das Ermüdungsverhalten von UHFB, Stahl-UHFB und Stahl-UHFB - Stahlbeton Plattenstreifen [24, 25].

\subsection{Nachweis der Gebrauchstauglichkeit}

Im Gebrauchszustand ist die UHFB Schicht ebenfalls unter Zugbeanspruchung mittragend. Die Spannungen, Verformungen und Steifigkeit des Verbundsystems werden mit dem Modell gemäß Bild 6 ermittelt. Dabei sind Eigenspannungen im Verbundquerschnitt infolge der Verformungsbehinderung des UHFB bei der Herstellung $\mathrm{zu}$ berücksichtigen. Mit diesem Modell wird beispielsweise nachgewiesen, dass eine Schicht aus UHFB flüssigkeitsdicht ist, falls im Grenzzustand der Gebrauchstauglichkeit sich der UHFB im verfestigenden Bereich befindet und die Zugdehnung das folgende Kriterium erfüllt [7]:

$\varepsilon_{U, \text { ser }} \leq 1 \%$

Dieses Kriterium impliziert eine rissefreie UHFB Schicht. 
4 4.1

\section{Anwendungen} Einleitung

Ein wichtiges Einsatzgebiet von UHFB ist die Verbesserung bestehender Betonbauten mit UHFB, indem die bekannten, immer wieder Schäden zeigenden Bauteilbereiche in Stahlbeton mit UHFB dauerhaft instandgesetzt werden. Seit 2004 wird UHFB in der Schweiz für die Verbesserung bestehender Betonbauten eingesetzt. Bei den nachfolgend kurz beschriebenen Anwendungen wurden verschiedene UHFB Mischungen eingesetzt, die sich je nach Anforderungen an die Verarbeitbarkeit und mechanischen Eigenschaften im Zementgehalt, Fasergehalt und Art des Hochleistungsverflüssigers unterschieden. Die Betonoberfläche des instandzusetzenden Bauteils wurde jeweils mit Hochdruckwasserstrahlen (HDW) und in einem Fall mit Sandstrahlen unter Einhaltung der bei der herkömmlichen Betoninstandsetzung geltenden Anforderungen an die Oberflächenrauigkeit vorbereitet. Damit konnte (ohne mechanische Verbindungselemente) ein genügender Verbund für ein monolithisches Tragverhalten der UHFB - Stahlbeton Verbundbauteile erhalten werden.

Bei allen Anwendungen wurden Vorversuche durchgeführt, um den UHFB mit den im jeweiligen Anwendungsfall zur Verfügung stehenden Personen, Geräten und anderen Gegebenheiten einwandfrei herstellen und einbauen zu können. Die Durchführung dieser Vorversuche war in allen Fällen wichtig, denn es konnten letzte Korrekturen vorgenommen werden, die danach eine problemlos ablaufende Ausführung mit der geforderten Qualität ermöglichten. Der Frisch-UHFB wurde bei allen Anwendungen ohne nennenswerte Schwierigkeiten in herkömmlichen Betonmischern auf der Baustelle oder im Betonwerk hergestellt und mit üblicher Gerätschaft (bei extremsten Umgebungstemperaturen von $4{ }^{\circ} \mathrm{C}$ respektive $33^{\circ} \mathrm{C}$ ) eingebaut. Die Dauer der Baustelle konnte in der Regel deutlich kürzer gehalten werden als bei herkömmlichen Methoden.

Die bisherigen Anwendungen können in drei Gruppen eingeteilt werden, welche die Verwendung von UHFB und Stahl-UHFB für die Herstellung der 1) Schutzfunktion auf horizontalen Flächen, 2) Schutzfunktion auf vertikalen Flächen, und 3) Trag- und Schutzfunktion für Platten betreffen.

\subsection{UHFB für die Herstellung der Schutzfunktion auf horizontalen Flächen}

Bei mehreren Anwendungen wurden die Oberflächen der Fahrbahnplatte und die Randabschlüsse von Brücken gemäß dem im Bild 7 dargestellten Konzept mit UHFB abgedichtet. Das Ziel der Instandsetzungen war die Wiederherstellung und Verbesserung der Dauerhaftigkeit. Diese Anwendungen beinhalteten viele der typischen Problemstellungen einer Instandsetzung von Straßenbrücken aus Stahlbeton: durch Chloride verursachte Bewehrungskorrosion der Randabschlüsse und der Oberseite der Fahrbahnplatte, eine Verbreiterung der Fahrbahn, und die Ar-

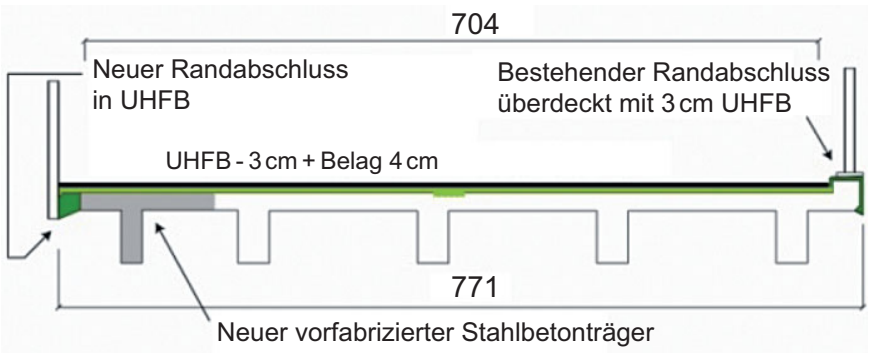

Bild 7 Querschnitt eines mit UHFB instandgesetzten und verbreiterten Plattenbalkens einer Brücke (Abmessungen in cm) [26] Cross section of a slab girder bridge that was rehabilitated and widened using UHPFRC (dimensions in $\mathrm{cm}$ ) [26]

beiten mussten unter Aufrechterhaltung des Straßenverkehrs ausgeführt werden.

Der UHFB wurde entsprechend der Grundidee in denjenigen Bereichen des Bauwerks eingesetzt, welche neu mit einer „Oberflächenhärtung“ den höchsten Beanspruchungen der Expositionsklassen XD2, XD3 dauerhaft standhalten müssen. Es wurden mit dem selbstverdichtenden UHFB Gefälle bis maximal 10,4\% hergestellt (Bild 8b). Dazu war eine angepasste Mischung notwendig, die ein thixotropes Verhalten des Frisch-UHFB ermöglicht, d. h. zäh fließend bei der Bearbeitung und starr an Ort bleibend nach dem Einbau. Oberflächen in SichtUHFB wurden roh geschalt oder in einem Fall mit einer profilierten Folie bearbeitet [27], um die Bildung von Rostpunkten an der mit chloridhaltigem Wasser beaufschlagten UHFB Oberfläche zu reduzieren und hervorstehende Fasern zu vermeiden (Bild 9).

In weiteren Anwendungen wurden Fahrbahnplatten mehrerer Straßenbrücken und einer Bahnbrücke mit UHFB abgedichtet resp. geschützt. Dabei standen folgende Argumente für den Einsatz von UHFB im Vordergrund: Reduktion der Dauer der Baustelle, Verlängerung der Wirksamkeit des Oberflächenschutzes, Vermeidung von Anpassungen vor/nach der Brücke, Reduktion von Bau- und Nutzerkosten, verbesserte Wirtschaftlichkeit.

\subsection{UHFB für die Herstellung der Schutzfunktion auf vertikalen Flächen}

Bei diesen Anwendungen wurde eine UHFB-Schicht in vertikaler Richtung auf bestehende wandartige Bauteile aufgebracht mit dem Ziel, eine langfristige Schutzfunktion zu erreichen. Im September 2006 wurden die Leitmauern einer Autobahnbrücke mit einer $30 \mathrm{~mm}$ dicken UHFB-Schicht beschichtet, um eine möglichst dauerhafte Leitmauer zu erhalten, denn künftige Instandsetzungen von herkömmlichen Schutzsystemen hätten sehr hohe Nutzerkosten zur Folge [28]. Die Herausforderung bestand darin, den selbstverdichtenden Frisch-UHFB in der richtigen Konsistenz in den $30 \mathrm{~mm}$ schmalen Hohlraum zwischen Schalung und der mit HDW vorbereiteten Oberfläche der Leitmauer über eine Höhe von rund $120 \mathrm{~cm}$ einzubringen (Bild 10a und 10b). Die UHFB- 

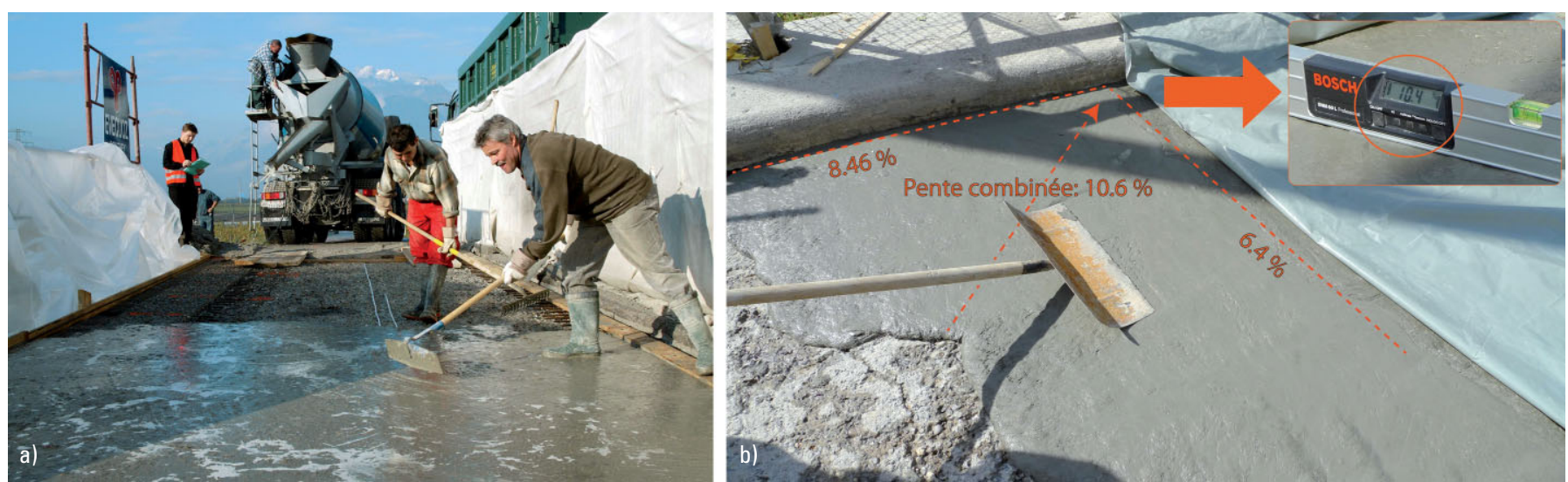

Bild 8 a) Einbau des Frisch-UHFB auf der Fahrbahnplatte, b) Herstellung eines Gefälles von 10,4 \% auf einer Brückenfahrbahnplatte a) Casting of fresh UHPFRC on a bridge deck slab, b) realization of a slope of $10.4 \%$ on a bridge deck slab
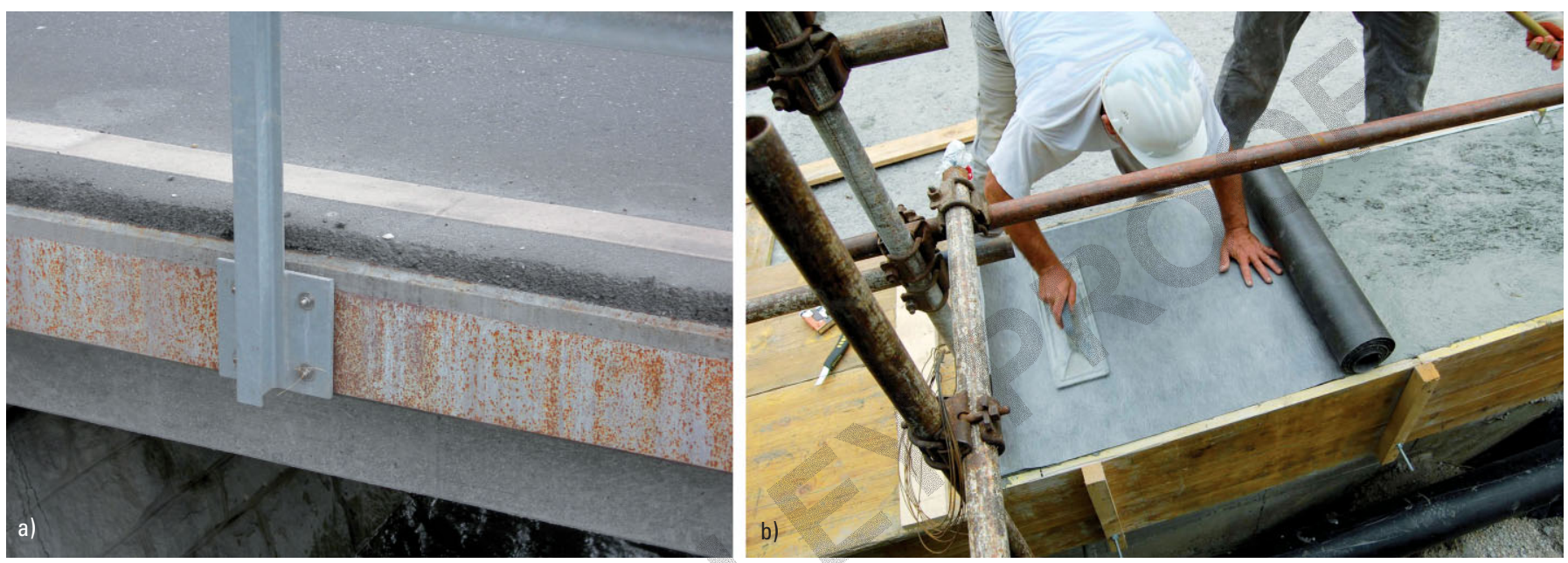

Bild 9 a) Rostpunkte auf einer in einer Stahlschalung hergestellten UHFB Oberfläche, b) Bearbeitung der Oberflächen mit profilierter Folie [27] a) corrosion stains on a UHPFRC casted against a steel formwork, b/ surface treatment of UHPFRC using a profiled membrane [27]
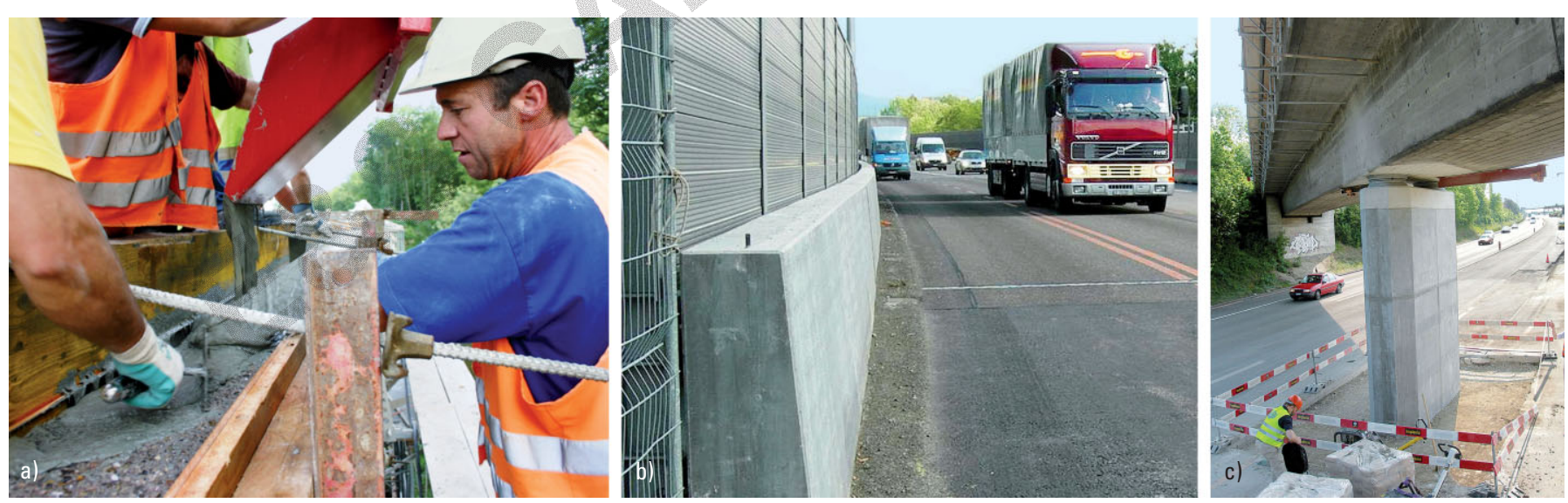

Bild 10 a) Einbringen des Frisch-UHFB, b) Ansicht der Leitmauer mit UHFB Beschichtung, c) UHFB-Fertigteile ummanteln einen Brückenpfeiler a) casting of fresh UHPFRC, b) view of the crash barrier wall covered with UHPFRC layer, c) prefabricated UHPFRC shell elements as protection shield for a bridge pier

Schicht wurde über die Leitmauer bis einige Zentimeter auf die Rückseite gezogen, um eine „Hinterläufigkeit“ in der kalten Fuge zu vermeiden.

Im Frühjahr 2007 wurde ein durch chloridinduzierte Bewehrungskorrosion stark beschädigter Brückenpfeiler einer Bahnüberführung mitten in einer stark befahrenen Autobahn instandgesetzt, indem der Pfeiler mit vorgefer- tigten Elementen aus UHFB mit einem dauerhaften Schutzschild gegen das Eindringen von Chloriden und Wasser geschützt wurde. Wegen der begrenzten Zugänglichkeit des Brückenpfeilers erwies sich die Fertigteilbauweise als vorteilhaft. Nach dem HDW-Abtrag des mit Chloriden verseuchten Betons wurden die einzelnen, $40 \mathrm{~mm}$ dünnen und bis zu $4 \mathrm{~m}$ hohen UHFB-Panels montiert und mit einem Zwei-Komponenten-Epoxy-Kleber 
verbunden; der Hohlraum hinter dem „UHFB-Schild“ und dem bestehenden Pfeiler wurde mit einem selbstverdichtenden Mörtel verfüllt (Bild 10c).

\subsection{Herstellung der erforderlichen Trag- und Schutzfunktion für Platten und Decken}

Die Stahlbetondecke der Fahrzeughalle in einem Industriegebäude wies eine unzureichende Tragfähigkeit für die kommende Generation von Fahrzeugen auf, worauf der Ersatz der bestehenden Decke vorgesehen wurde. Eine in den Baukosten deutlich günstigere Unternehmervariante wurde ausgeführt, indem die bestehenden Rippendecke mit einer rund $40 \mathrm{~mm}$ starken Stahl-UHFB Schicht verstärkt wurde, die anstelle des zuvor vorhandenen Überzugs aufgebracht wurde (Bild 11). Diese bauliche Maßnahme erwies sich zudem in Bezug auf die Nutzerkosten als vorteilhaft, indem die Nutzung des Gebäudes während der Bauarbeiten relativ wenig beeinträchtigt wurde. Zudem waren die Bauzeit kürzer und damit die Lärmund Staubentwicklung geringer.

Im Falle einer Instandsetzung und Verstärkung der Fahrbahnplatte einer Brücke von hohem kulturellem Wert, die dem lokalen Verkehr in einem Dorf dient, wurde eine Schicht aus Stahl-UHFB für die Wiederherstellung der Trag- und Schutzfunktion der Fahrbahnplatte aufgebracht (Bild 12a). Der frische UHFB wurde in einer nahe gelegenen Betonzentrale hergestellt, mit Lastwagen angeliefert und eingebaut. Es wurde eine direkt befahrbare UHFB-Fahrbahnfläche hergestellt, indem durch Einstreuen von Splittkies eine für den gegebenen Verkehr genügende Rauigkeit der Oberfläche erzielt werden konnte. Damit erfüllt die Stahl-UHFB Schicht drei Aufgaben: Steigerung des Tragwiderstands, Abdichtung der Fahrbahnplatte, befahrbare Deckschicht der Fahrbahnplatte.

In einer im Jahre 2011 ausgeführten Anwendung wurde die massive Platte einer Straßenbrücke, die von Einzelstützen abgefangen wird, mit Stahl-UHFB verstärkt, um die erforderliche Schub- und Biegetragfähigkeit über den Stützen herzustellen (Bild 12b).

\subsection{Kosten und Wirtschaftlichkeit}

In der Konzeptphase eines Einsatzes der Stahl-UHFB Stahlbeton Verbundbauweise werden die Kosten und die Wirtschaftlichkeit der Bauweise auf vier unterschiedlichen Stufen betrachtet, was nachfolgend am Beispiel eines Projekts für einen Eingriff in bestehende Tragwerke erläutert wird:

- 1 Baustoffkosten: Die Baustoffkosten für UHFB liegen zwischen denjenigen von Baustahl und Stahlbeton. Der Preis für die Herstellung und Lieferung von UHFB für die Ausführung einer $30 \mathrm{~mm}$ dicken Schicht auf einer Brückenfahrbahnplatte beträgt aufgrund heutiger Erfahrungen 120 bis 180 Euro pro $\mathrm{m}^{2}$.

- 2 Baukosten: Die direkten Baukosten für eine Instandsetzung mit UHFB können im Rahmen der Kosten für heute (noch) übliche Bauweisen gehalten werden, denn die UHFB Bauweise ermöglicht einen rationelleren Bauvorgang. Indem mehrere Anforderungen und Funktionen mit einem Arbeitsgang realisiert werden, werden die Anzahl der Arbeitsschritte und -stunden sowie die Dauer der Baustelle reduziert. Bei einigen Anwendungen konnten die Baukosten der UHFB Bauweise im Vergleich zu den herkömmlichen Methoden deutlich geringer gehalten werden.

- 3 Gesamtkosten: Durch eine kürzere Dauer der Baustelle werden die aus volkswirtschaftlicher Sicht immer bedeutender werdenden Nutzerkosten reduziert. Die Wirtschaftlichkeit einer Baumaßnahme kann so deutlich erhöht werden.

- 4 Lebenszykluskosten: Auch über die Nutzungsdauer betrachtet dürfte sich der Einsatz von UHFB gegenüber herkömmlichen Methoden als wirtschaftlich erweisen, denn es darf für das mit UHFB qualitativ verbesserte Bauwerk mit einem geringeren Aufwand für den Unterhalt und die Instandsetzung gerechnet werden. Auch kann abgeschätzt werden, dass mit dieser neuartigen Bauweise der Ressourcenverbrauch verringert wird.

Entscheidend für den Einsatz der Stahl-UHFB - Stahlbeton Verbundbauweise sind in Wirklichkeit immer die
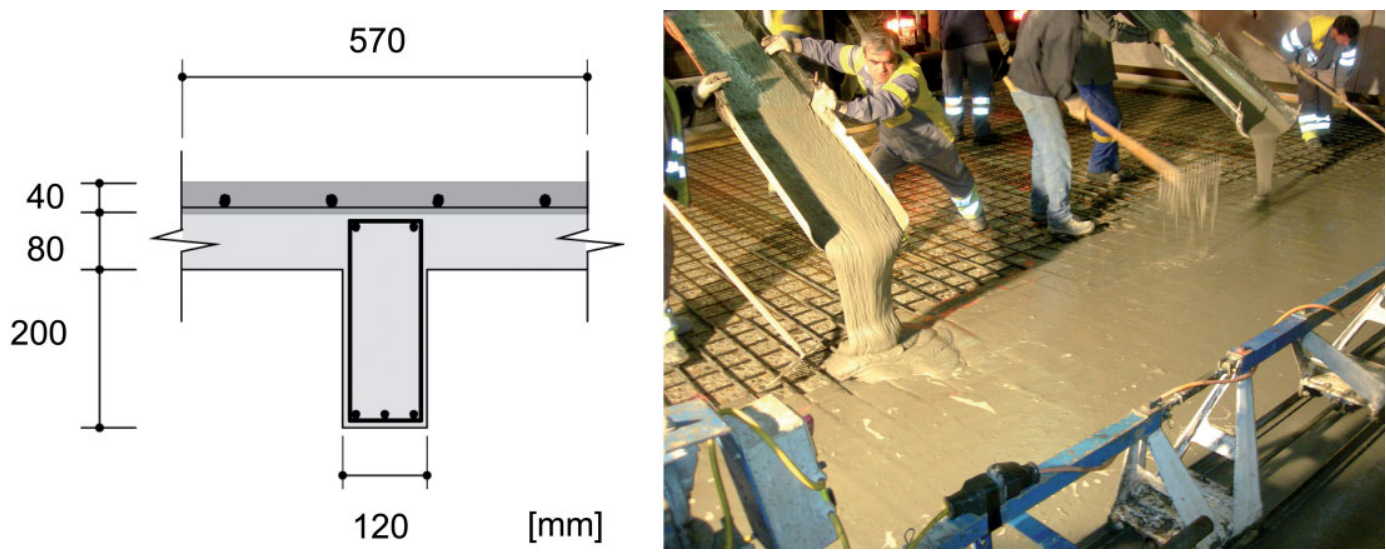

Bild 11 Querschnitt über Auflager der mit bewehrtem Stahl-UHFB verstärkten Rippendecke und Einbringen des Frisch-UHFB Cross section over support of a ribbed slab strengthened with R-UHPFRC and casting of fresh UHPFRC 

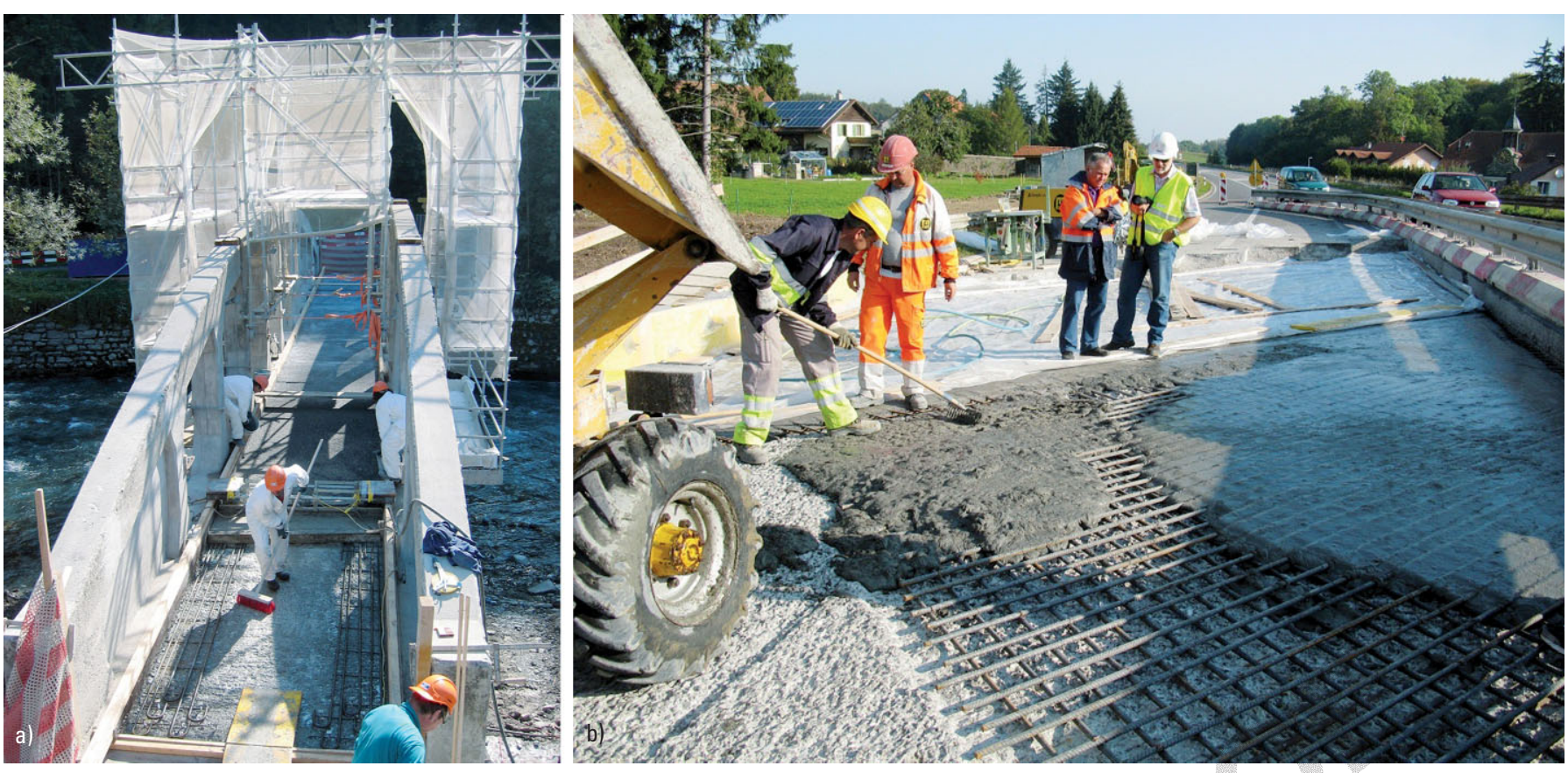

Bild 12 Trag- und Schutzfunktion durch Stahl-UHFB: a) Fahrbahnplatte als Zuggurt einer Bogenbrücke, b) Biege- und Schub-Verstärkung über Stützen einer massiven Platte

Load carrying and protection function using R-UHPFRC: a) deck slab as tensile member of an arch bridge, b) bending and shear strengthening of a massive slab over a column

Baukosten, also die Stufe 2, denn die heute noch angewendeten Finanzierungsmechanismen im Bauwesen berücksichtigen die Stufen 3 und 4 nach wie vor nicht explizit. Somit besteht bei einer UHFB Anwendung aus Sicht der Kosten das Ziel darin, bereits auf der Stufe 2 der direkten Baukosten gegenüber der herkömmlichen Bauweise kompetitiv, d.h. kostengünstiger, zu sein. Denn man kann nicht davon auszugehen, dass die Budgets für teurere, langfristig jedoch wirtschaftlichere Baumaßnahmen jemals erhöht werden. Geringere Nutzerkosten und Lebenszykluskosten werden üblicherweise nur bei gleichen Baukosten als qualitatives Entscheidungskriterium berücksichtigt.

\subsection{Nachhaltigkeit}

Betrachtungen zur Umweltverträglichkeit von Baumethoden sind für moderne (öffentliche) Bauherr- und Bauwerkseigentümerschaften heute selbstverständlich. UHFB wird zwar mit vergleichsweise hohen Zementmengen hergestellt, doch kann die geforderte technische Leistungsfähigkeit mit relativ dünnen UHFB Schichtstärken erreicht werden. Es kann somit abgeschätzt werden, dass insgesamt gegenüber der herkömmlichen Betonbauweise die verwendete Zementmenge nicht größer wird, die Kiesreserven jedoch stark geschont werden.

Auch muss weniger Baustoffvolumen hergestellt und weniger Baustoffmasse transportiert werden. Somit sind tendenzmäßig der Energieaufwand und der $\mathrm{CO}_{2}$-Ausstoß insgesamt geringer als bei herkömmlichen Bauweisen. Durch den schonenderen Umgang mit bestehender Bausubstanz, welcher der UHFB-Technologie zugrunde liegt, können zudem vor allem bei der Erhaltung von Betonbauten die Rückbaumenge und somit der Bedarf an Deponien und Recycling vermindert werden.

Beim „Bauen im Bestand“ lautet das strategische Ziel, die Bauwerke derart zu verbessern, dass der weitere Aufwand für deren Erhaltung langfristig drastisch gesenkt werden kann. Dadurch sollen Geldmittel für Investitionen in neue, den Regeln der Nachhaltigkeit genügende Bausubstanz für Hochbauten und Infrastrukturbauwerke mit einem echten volkswirtschaftlichen Mehrwert frei werden. Dieses strategische Ziel erfordert Interesse, Offenheit und Engagement für neuartige Technologien und deren Anwendung.

\section{$5 \quad$ Weitere Anwendungsmöglichkeiten}

Die Eigenschaften von Stahl-UHFB bieten weitere Anwendungsmöglichkeiten. Nachfolgend werden ein paar Konzeptideen beschrieben:

- Fertigteile für Bruickenkappen und Randabschliusse: Die dem Spritzwasser ausgesetzten Randbereiche vieler Straßenbrücken weisen frühzeitig Schäden in Form von Bewehrungskorrosion auf. Eine Instandsetzungsmethode besteht darin, die beschädigten Randbereiche abzutrennen und durch UHFB Fertigteile (mit entsprechend reduzierten Abmessungen) zu ersetzen.

- Schließen von Dilatationsfugen: Die meisten bestehenden Fugen sind wasserdurchlässig, und der dadurch mit dem chloridhaltigen Wasser in Kontakt stehende Stahlbeton weist entsprechend frühzeitige 
(Korrosions-)Schäden auf. Heute eingebaute Systeme für Fahrbahnübergänge sind zudem wenig zuverlässig in Bezug auf die Flüssigkeitsdichtigkeit. Gerade bei Gerbergelenken ergeben sich dadurch gefährliche Situationen. Die meisten dieser Fugen bestehender Brücken könnten geschlossen werden, da die Schwindund Kriechverformungen der Betonkonstruktion weitgehend abgeklungen sind. Die Idee besteht somit darin, zunächst die Bewehrung der beiden zu verbindenden Teile freizulegen und das Detail mit UHFB ergänzt mit entsprechenden Bewehrungszulagen zu verschließen. Dadurch entsteht ein integrales (fugenloses) Bauwerk.

- Es ist naheliegend, den Einsatz von Stahl-UHFB jeweils in Situation zu prüfen, bei denen hohe konzentrierte Kräfte eingeleitet werden müssen wie z. B. Ankerblöcke, Verankerungen, Knoten, Auflager.

- Der Ersatz oder die Verbreiterung der Fahrbahnplatte von Brücken kann mithilfe von Fertigteilen ausgeführt werden, die in Längsrichtung mit Vorspannkabeln zusammengespannt und deren in Querrichtung verlaufende Fugen verklebt werden. Die Fertigteile für die Fahrbahn werden dabei bei deren Herstellung mit einer Schicht UHFB oder Stahl-UHFB als bereits eingebaute Abdichtung versehen. Diese Bauweise ermöglicht einen schnellen Bauvorgang mit einer entsprechend kurzen Behinderung der Nutzung der Brücke. Auch ist denkbar, diese Fertigteile aus Stahl-UHFB in Leichtbauweise herzustellen.

- UHFB kann in der Fertigteilbauweise verwendet werden, um Ausristungsteile wie Randsteine oder Radresp. Fahrzeug-abweisende Elemente herzustellen.

Diese Konzeptideen sind nicht auf bestehende Bauwerke begrenzt. Sie können analog auch im Neubau als Mischbauweise umgesetzt werden in Form von Tragwerken in Verbundbauweise aus Stahl-UHFB - Stahlbeton [29], aus Stahl-UHFB - Holz oder aus Stahl-UHFB - Stahl. Dabei ergeben sich neuartige Konstruktionsweisen, die gegenüber der etablierten, herkömmlichen Verbundbauweise Vorteile bieten können.

\section{Regelwerke}

Eine wichtige Voraussetzung für die breite Anwendung einer neuartigen Technologie ist ein Regelwerk und damit die Anerkennung als „Stand der Baukunde“ [30, 31]. Ein Regelwerk würde zudem die Anwendung einer neuartigen Technologie durch die Projektierenden und Ausführenden erleichtern. Auf internationaler Ebene sind vor allem die Empfehlungen der AFGC [32] und des JSCC [33] bekannt. Die fib Task Group 8.6 erarbeitet ebenfalls Empfehlungen für den Neubau mit UHFB [34], welche allerdings einzig neue Tragwerke in UHFB Bauweise behandeln.

In der Schweiz ist momentan ein Regelwerk unter dem Titel „Ultra-Hochleistungs-Faserbeton (UHFB) - Baustoffe, Bemessung und Ausführung“ in Bearbeitung. Dieses Regelwerk behandelt sowohl die Stahl-UHFB - Stahlbeton Verbundbauweise als auch die Stahl-UHFB Bauweise für neuartige Tragwerke in Leichtbauweise.

\section{$7 \quad$ Folgerungen}

Dieser Aufsatz fasst die wesentlichen Erkenntnisse zusammen beim Einsatz von Ultra-Hochleistungs-Faserbeton (UHFB) zur Verstärkung von bestehenden Stahlbetonbauteilen. Durch das Aufbringen einer vergleichsweise dünnen Schicht aus Ultra-Hochleistungs-Faserbeton (UHFB) werden Bauteile aus Stahlbeton effizient verstärkt, indem der Tragwiderstand im Grenzzustand der Tragfähigkeit erhöht, das Tragverhalten im Gebrauchszustand verbessert sowie die Dauerhaftigkeit verlängert werden. Dabei ist es vorteilhaft, in die Haupttragrichtung Betonstahlstäbe in die UHFB Schicht einzulegen. Die Anwendungen dieser neuartigen Technologie unter Baustellenbedingungen zeigen, dass die UHFB Technologie bereits regelmäßig angewendet wird und für eine breite Anwendung reif ist.

\section{Dank}

Die Autoren danken allen Doktoranden, Studenten und Gastforschern, die seit 1999 in der „UHFB-Gruppe“ am MCS-Lehrstuhl an der ETH Lausanne (EPFL) zum Inhalt dieses Aufsatzes beigetragen haben. Dank gebührt auch verschiedenen Institutionen, welche die bisherigen Forschungen am MCS-EPFL finanziell unterstützt haben, sowie bei mehreren Bauwerkseigentümern, Projektingenieuren, Unternehmern und Betonherstellern, die bei den Anwendungen beteiligt waren.

\section{Literatur}

[1] WueST, J.: Comportement structural des bétons de fibres ultra performants en traction dans les éléments composés. Thèse de doctorat No 3987, Ecole Polytechnique Fédérale de Lausanne EPFL, 2007.

[2] DENARIÉ, E.: Recommendations for the tailoring of UHPFRC recipes for rehabilitation, Deliverable D06, European Research Project ARCHES - Assessment and Rehabilitation of Central European Highway Structures, November 2009.

[3] Wuest, J.; DENARIÉ, E.; BRÜHWILER, E.: Model for predicting the UHPFRC tensile hardening response. Proceedings, The
Second International Symposium on Ultra High Performance Concrete, March 05 - 07, 2008, Kassel, Germany.

[4] BrÜHWiler, E.; DenARIÉ, E.; OesterleE, C.: Hochleistungsfähiger Faserfeinkornbeton zur Effizienzsteigerung bei der Erhaltung von Kunstbauten aus Stahlbeton. VSS Bericht, Forschungsauftrag AGB 2005/004, VSS Zürich, Januar 2010.

[5] Torrent, R.; Denarié, E.; Jacobs, F.; Leemann, A.; TERUZZI, T.: Specification and site control of the permeability of the cover concrete: the Swiss approach. Materials and Corrosion, in press. 
[6] ŠAjNA, A.; DenARIÉ, E.; Bras, V.: Assessment of a UHPFRC based bridge rehabilitation in Slovenia, two years after application. HIPERMAT Conference, Kassel, March 7-9 2012.

[7] Charron, J.-P.; Denarié, E.; BrÜHWiler, E.: Permeability of Ultra High Performance Fiber Reinforced Concretes (UHPFRC) under high stresses. Materials and Structures, Vol. 40, No 3 (March), 2007, pp. 269-277.

[8] DenarIÉ, E.; BRÜHWILER, E.: Strain Hardening of Ultra-high Performance Fibre Reinforced Concrete: Deformability versus Strength Optimization. International Journal of Restoration of Buildings and Monuments, Aedificatio Publishers Freiburg Germany, Vol. 17, No. 6, 2011, pp. 397-410.

[9] OesterleE, C.; BRÜHWILER, E.; DenARIÉ, E.: Tragverhalten von Verbundbauteilen aus bewehrtem UHFB und Stahlbeton. Beton- und Stahlbetonbau 104 (2009), Heft 8, S. 462470.

[10] OesteRlEe, C.: Structural response of reinforced UHPFRC and RC composite members. Doctoral Thesis No. 4848, Ecole Polytechnique Fédérale de Lausanne EPFL, 2010.

[11] JUngwiRTH, J.: Zum Tragverhalten von zugbeanspruchten Bauteilen aus Ultra-Hochleistungs-Faserbeton. Dissertation EPFL No. 3429, Lausanne, Switzerland, 2006.

[12] LEUTBECHER, T.: Rissbildung und Zugtragverhalten von mit Stabstahl und Fasern bewehrtem Ultrahochfesten Beton (UHPC). Dissertation Universität Kassel, Kassel, Germany, 2007.

[13] Redaelli, D.: Comportement et modélisation des éléments de structure en béton fibré à ultra-hautes performances avec armatures passives. Thèse EPFL No 4298, Lausanne, Switzerland, 2009.

[14] Kamen, A.; Denarié, E.; SADOUKI, H.; BrÜHWILER, E.: Thermo-mechanical response of UHPFRC at early age - experimental study and numerical simulation. Cement and Concrete Research, Vol. 38, Issue 6, June 2008, pp. 822-831.

[15] Habel K.; Charron J. P.; Denarié E.; BrÜHWILER E.: Autogenous deformations and viscoelasticity of UHPFRC in structure. Magazine of Concrete Research, 58, No 3, 2006, pp.135156.

[16] HABEL, K.; DeNARIÉ, E.; BRÜHWILER, E.: Structural response of elements combining Ultra-High Performance Fiber Reinforced Concretes and reinforced concrete. Journal of Structural Engineering, ASCE, Vol. 132, No. 11, 2006, pp. 1793-1800.

[17] Habel K.; Denarié E.; BRÜHWILER E.: Experimental Investigation of Composite Ultra-High-Performance Fiber-Reinforced Concrete and Conventional Concrete Members. ACI Structural Journal, Vol. 104, No 1, 2007, pp. 93-101.

[18] HABEL, K.; DENARIÉ, E.; BRÜHWILER, E.: Bauteile aus Ultrahochleistungsfähigem Faserbeton (UHPFRC) und traditionellem Stahlbeton - Eine innovative Lösung zur Instandsetzung und Veränderung bestehender Betonbauten. Beton- und Stahlbetonbau 100 (2005), Heft 2, S. 124-131.

[19] Alaee, F. J.; Karihaloo, B. L.: Retrofitting of Reinforced Concrete Beams with CARDIFRC. Journal of Composites for Construction, 7 (3, 2003): pp. 174-186.

[20] Kamen, A.; Denarié, E.; SAdouki, H.; BrÜHWILER, E.: Numerical simulations of early age thermal effects. Cement and Concrete Research, Vol. 38, Issue 6, 2008, pp. 822-831.

[21] Oesterlee, C.; SAdOUKI, H.; BrÜHWILER, E.: FE analysis of a composite bridge structure combining UHPFRC and prestressed concrete. Proceedings, UHPC-2008: The Second International Symposium on Ultra High Performance Concrete, March 05-07, 2008, Kassel, Germany.

[22] NOSHIRAVANI, T.; BRÜHWILER, E.: Experimental Investigation on R-UHPFRC - RC Composite Beams Subjected to Combined Bending and Shear. ACI Structural Journal. (in press)

[23] Noshiravani, T.; BrÜHWIler, E.: Analytical Model for Predicting the Response and Flexure-Shear Resistance of
R-UHPFRC - RC Composite Beams. ASCE Journal of Structural Engineering, accepted for publication.

[24] MAKITA, T.; BRÜHWILER, E.: Tensile fatigue behaviour of Ultra-High Performance Fibre Reinforced Concrete. Materials and Structures, accepted for publication.

[25] MAKITA, T.; BRÜHWILER, E.: Fatigue behaviour of bridge deck slab elements strengthened with reinforced UHPFRC. IABMAS 2012, Stresa, Italy, July 8-12, 2012.

[26] BRÜHWILeR, E.; Denarié, E.; PutAllaZ, J.-C.: Instandsetzung einer Betonbrücke mit Ultrahochleistungsfähigem Faserfeinkornbeton (UHLFB). Beton- und Stahlbetonbau 100 (2005), Heft 8, S. 822-827.

[27] DENARIÉ, E.: Recommendations for the use of UHPFRC in composite structural members. Deliverable D14, European Research Project ARCHES - Assessment and Rehabilitation of Central European Highway Structures, November 2009.

[28] Oesterlee, C.; Denarié, E.; BRÜHWILER, E.: In-situ casting of UHPFRC protection layer on crash barrier walls. Proceedings, Advances in Construction Materials - Symposium in honour of HANS W. REINHARDT: July 23-24, 2007, University of Stuttgart, Germany.

[29] BrÜHWiler, E.; Fehling, E.; Bunje, K.; Pelke, E.: Design of an innovative composite road bridge combining reinforced concrete with Ultra-High Performance Fibre Reinforced Concrete. Proceedings, IABSE Symposium "Improving Infrastructure Worldwide", Weimar, September 2007.

[30] Deutscher Ausschuss für Stahlbeton, Heft 561, Sachstandsbericht Ultrahochfester Beton, Beuth-Verlag, Berlin, 2008.

[31] AFGC/SETRA, Bétons Fibrés à Ultra Hautes Performances, Recommandations provisoires, éditées par Association Française de Génie Civil et Service Technique des Routes et des Autoroutes, ref. SETRA: F0211 (in French and English) (2002)

[32] Japan Society of Civil Engineers, Recommendations for design and construction of ultra-high strength fiber reinforced concrete structures, JSCE Guidelines for Concrete No 9, September 2009.

[33] fib Recommendations for UHPFRC, Fédération Internationale du Beton - fib Task Group 8.6: "Ultra High Performance Fibre Reinforced Concrete - UHPFRC" (work in progress).

[34] BRÜHWILER, E.; MoreIllon, L.; Suter, R. (Herausgeber): Ultra-Hochleistungs-Faserbeton - entwerfen, bemessen, bauen: Tagungsband der 1. Schweizer UHFB Konferenz, Fachhochschule Fribourg vom 27. Oktober 2011, ISBN 9782-8399-0951-8, 175 p (auf Deutsch und Französisch).

Autoren

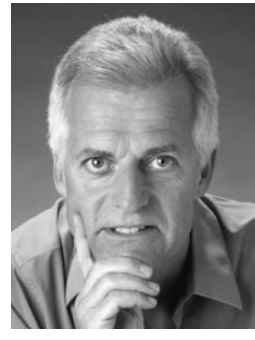

Prof. Dr. Dipl.-Bauing. ETH Eugen Brühwiler eugen.bruehwiler@epfl.ch

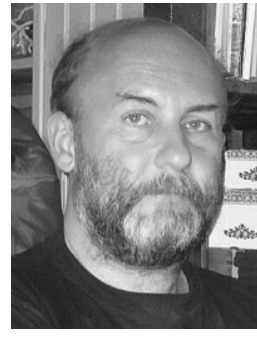

Dr. Dipl.-Bauing. ETH Emmanuel Denarié emmanuel.denarie@epfl.ch
Eidgenössische Technische Hochschule Lausanne (EPFL) Lehrstuhl für Erhaltung und Sicherheit von Bauwerken (MSC) Station 18

1015 Lausanne, Schweiz 